\title{
Normal-state properties of a resonantly interacting $p$-wave Fermi gas
}

\author{
Juan Yao ${ }^{1,2}$ and Shizhong Zhang ${ }^{1, *}$ \\ ${ }^{1}$ Department of Physics and Centre of Theoretical and Computational Physics, The University of Hong Kong, Hong Kong, China \\ ${ }^{2}$ Institute for Advanced Study, Tsinghua University, Beijing 100084, China
}

(Received 26 February 2018; published 13 April 2018)

\begin{abstract}
Motivated by the recent experimental progress in the study of $p$-wave resonant Fermi gases, we investigate the normal-state properties of such a gas. We calculate the universal equation of state and the two $p$-wave contacts that characterize the universal properties of the system, in good agreement with experiments. Furthermore our calculation obtains the superfluid transition temperature $T_{c}$ within the Nozières and Schmitt-Rink (NSR) scheme and determines the analytic expressions for $T_{c}$ in the weak-coupling limit. We show explicitly the nonperturbative nature of the effective range corrections.
\end{abstract}

DOI: 10.1103/PhysRevA.97.043612

\section{INTRODUCTION}

Studies of $p$-wave Feshbach resonances with ultracold atomic gases date back to 2003 when they were first observed in ${ }^{40} \mathrm{~K}$ [1]. This and subsequent experiments [2-11] have explored the collisional properties of the gas in detail and showed that the system suffers significant loss close to resonance. In contrast, broad $s$-wave Feshbach resonances are stabilized by Pauli exclusion of three-body processes, allowing an s-wave resonant superfluid to be realized [12]. The difference between the $s$ - and $p$-wave resonances stems from the existence of centrifugal barrier in the case of $p$-wave resonance, which tends to support quasibound dimer states within the centrifugal barrier and this leads to significant atom loss, preventing the study of a resonant $p$-wave gas in equilibrium [5,13].

However, a recent experimental study [14] of a single component Fermi gas of ${ }^{40} \mathrm{~K}$, utilizing a fast spectroscopic measurement, has shown that close to the $p$-wave Feshbach resonance, the system can establish quasiequilibrium between the scattering fermions and the quasibound dimer states, while suffering an overall loss that still allows the study of properties of the gases to be conducted. In this way, it is demonstrated experimentally that the $p$-wave resonant Fermi gas obeys a set of universal relations controlled by the $p$-wave contacts [15-21], analogous to the $s$-wave case [22-25]. Unlike the $s$-wave case where usually a single parameter, the $s$-wave scattering length, is sufficient for the description of the universal properties, in the $p$-wave case, one needs to take into account the effective range corrections in order to formulate a consistent theory [16]. In the presence of externally or spontaneous broken axial rotation symmetry, the $p$-wave contacts have to be extended to a tensor [26,27] (see also Ref. [28]). Many-body physics with $p$-wave resonance has been explored extensively (for a review, see Ref. [29]), including the discussion of $p$-wave polaron [30]. So far, however, no explicit calculation of the $p$ contacts exists except via Virial expansion [16], leaving unexplored an exciting regime of $p$-wave resonant Fermi gases in the normal state, and in particular, close to the superfluid transition temperature.

*shizhong@hku.hk
In this article, we develop a many-body theory for the normal state of a single component $p$-wave Fermi gas. We adopt a two-channel formulation of $p$-wave resonances and extend the Nozières and Schmitt-Rink (NSR) scheme for $s$-wave resonances [31], emphasizing the role of $p$-wave effective range. We calculate the universal equation of state for the resonant Fermi gases in the normal state and also the associated $p$-wave contacts [16]. Finally we determine the superfluid transition temperature $T_{c}$ of a $p$-wave Fermi gas, using parameters appropriate to the current experiment. An analytic expression for $T_{c}$ is also obtained in the weak-coupling limit that shows explicitly its nonperturbative dependence on the effective range, and further emphasizes its special importance as compared with the $s$-wave case.

\section{TWO-CHANNEL MODEL}

We adopt a two-channel description of the $p$-wave Feshbach resonance for a spinless Fermi gas where the Feshbach resonance is generated by including explicitly a closed-channel molecular field corresponding to the $p$-wave bound state. The noninteracting Hamiltonian

$$
\hat{H}_{0}=\sum_{\mathbf{k}} \epsilon_{\mathbf{k}} \hat{a}_{\mathbf{k}}^{\dagger} \hat{a}_{\mathbf{k}}+\sum_{m, \mathbf{q}}\left(\epsilon_{\mathbf{q}} / 2-v_{m}\right) \hat{b}_{m, \mathbf{q}}^{\dagger} \hat{b}_{m, \mathbf{q}},
$$

where $\hat{a}_{\mathbf{k}}^{\dagger}$ is the creation operator for (spinless) fermions with momentum $\hbar \mathbf{k}$ with kinetic energy $\epsilon_{\mathbf{k}}=\hbar^{2} \mathbf{k}^{2} / 2 M$, where $M$ is the fermion mass. $\hat{b}_{m, \mathbf{q}}^{\dagger}$ is the creation operator for closedchannel molecules with momentum $\hbar \mathbf{q}$ and relative angular momentum projection $m$. In this work, we work close to a $p$ wave resonance and neglect other partial wave scatterings. As a result, $m=0, \pm 1$. In actual experiment for ${ }^{40} \mathrm{~K}$, the resonances for $m=0$ and $m= \pm 1$ are split by about $0.5 \mathrm{G}[1,14]$. This is taken into account by assuming an $m$-dependent detuning $v_{m}$ of the closed-channel molecules. The conversion between the open channel scattering fermions and the closed-channel molecules is given by

$$
\hat{V}=\sum_{m, \mathbf{k}, \mathbf{q}} \frac{g_{m}}{\sqrt{2 V}} k Y_{1 m}(\hat{\mathbf{k}}) \hat{a}_{\frac{\mathbf{q}}{2}-\mathbf{k}}^{\dagger} \hat{a}_{\frac{\mathbf{q}}{2}+\mathbf{k}}^{\dagger} \hat{b}_{m, \mathbf{q}}+\text { H.c. }
$$


where $g_{m}$ is the coupling constant. The matrix element $k Y_{1 m}(\hat{\mathbf{k}})$ arises from the $p$-wave symmetry of the molecules where $k=$ $|\mathbf{k}|$ and $\hat{\mathbf{k}}=\mathbf{k} / k$. The bare coupling constants $\left\{v_{m}, g_{m}\right\}$ can be related to the low-energy $p$-wave scattering parameters by a standard renormalization procedure (hereafter we set $\hbar=1$ ) $[14,15,29]$

$$
\begin{aligned}
v_{m}^{-1} & =\frac{4 \pi v_{m}}{g_{m}^{2} M}+\frac{4 \pi}{V} \sum_{\mathbf{k}} 1, \\
R_{m}^{-1} & =\frac{4 \pi}{g_{m}^{2} M^{2}}+\frac{2 \pi}{M V} \sum_{\mathbf{k}} \frac{1}{\epsilon_{\mathbf{k}}},
\end{aligned}
$$

where we have introduced the $p$-wave scattering volume $v_{m}$ and effective range $R_{m}$ through the low-energy effective range expansion

$$
k^{3} \cot \delta_{m}(k)=-\frac{1}{v_{m}}-\frac{1}{R_{m}} k^{2} .
$$

$\delta_{m}(k)$ is the $p$-wave scattering phase shift with magnetic projection $m$. Note that we have neglected the direct $p$-wave interaction between the spin-polarized fermions. The total number of fermions $N$ is given by $N=N_{\mathrm{F}}+2 N_{\mathrm{B}} \equiv N_{\mathrm{F}}+$ $2 \sum_{m} N_{\mathrm{B}, m}$, where $N_{\mathrm{F}}=\sum_{\mathbf{k}} a_{\mathbf{k}}^{\dagger} a_{\mathbf{k}}$ and $N_{\mathrm{B}, m}=\sum_{\mathbf{k}} b_{m, \mathbf{k}}^{\dagger} b_{m, \mathbf{k}}$. In the following, we define the Fermi wave vector $k_{F}$ by $n \equiv N / V=k_{F}^{3} /\left(6 \pi^{2}\right)$, where $n$ is the number density and $V$ is the volume of the system.

\section{NOZIÈRES AND SCHMITT-RINK SCHEME FOR $P$-WAVE GASES}

To describe the normal-state properties of a spinless Fermi gas with $p$-wave interactions, we adopt the standard Nozières and Schmitt-Rink scheme, extended to the $p$-wave channel. In the normal state, the thermodynamic potential $\Omega$ can be written as $\Omega=\Omega_{0}^{\mathrm{F}}+\Omega_{0}^{\mathrm{M}}+\Omega_{\text {int }}$, where $\Omega_{0}^{\mathrm{F}}=$ $-1 / \beta \sum_{\mathbf{k}} \ln \left[1+\exp \left(-\beta \xi_{\mathbf{k}}\right)\right]$ gives the contribution of noninteracting fermions. $\beta=1 / k_{\mathrm{B}} T$ and $\xi_{\mathbf{k}}=\epsilon_{\mathbf{k}}-\mu$ is the kinetic energy of fermions measured from its chemical potential $\mu . \Omega_{0}^{\mathrm{M}}=1 / \beta \sum_{m, \mathbf{q}} \ln \left(1-\exp \left[-\beta\left(\epsilon_{\mathbf{q}} / 2-2 \mu-v_{m}\right)\right]\right)$ gives the contribution from bosonic molecules. Note that, while $\Omega_{0}^{\mathrm{F}}$ depends only on physical parameters, the expression for $\Omega_{0}^{\mathrm{M}}$ involves the bare detuning $v_{m}$, which has to be renormalized later. Within NSR [31], the contribution to $\Omega$ from the interaction term is given by the ring diagrams in Fig. 1. Explicitly, we have

$$
\begin{aligned}
\Omega_{\mathrm{int}}= & \sum_{m, \mathbf{q}} \int_{-\infty}^{\infty} \frac{d z}{\pi} \frac{1}{e^{\beta z}-1} \\
& \times \operatorname{Im}\left\{\ln \left[1+g_{m}^{2} \Pi_{m}\left(\mathbf{q}, z+i 0^{+}\right) G_{0}^{\mathrm{M}}\left(\mathbf{q}, z+i 0^{+}\right)\right]\right\},
\end{aligned}
$$

where $G_{0}^{\mathrm{M}}\left(\mathbf{q}, i v_{n}\right)$ is the Green's function for noninteracting molecules,

$$
G_{0}^{\mathrm{M}}\left(\mathbf{q}, i v_{n}\right)=\frac{1}{i v_{n}-\left(\epsilon_{\mathbf{q}} / 2-v_{m}-2 \mu\right)},
$$

and $i v_{n}=2 \pi n i / \beta \hbar$ is the bosonic Matsubara frequency with integer $n$. The polarization $\Pi_{m}$ describes the propagation of

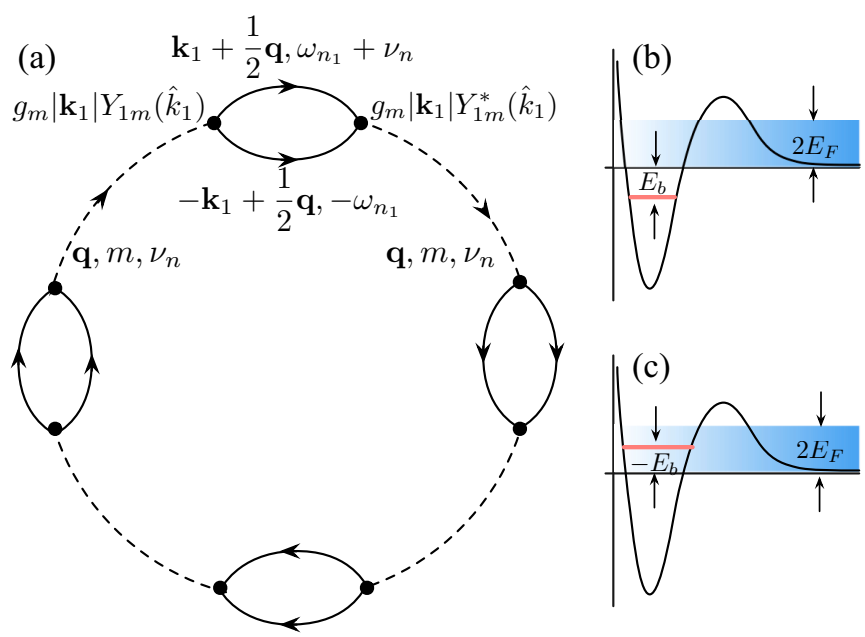

FIG. 1. (a) Diagrams that contribute to the thermodynamic potential in the Nozières and Schmitt-Rink scheme. The solid lines represent the Green's functions for fermions $G_{0}^{\mathrm{A}}\left(\mathbf{k}, i \omega_{n}\right)=\left[i \omega_{n}-\right.$ $\left.\left(\epsilon_{k}-\mu\right)\right]^{-1}$. The dashed lines represent the Green's function for molecules $G_{0}^{\mathrm{M}}\left(\mathbf{q}, i v_{n}\right)$ of Eq. (7). The vertex is given by $g_{m}\left|\mathbf{k}_{1}\right| Y_{1 m}\left(\hat{k}_{1}\right)$, indicating the $p$-wave scattering through channel $m$. (b) Schematic diagrams of an actual bound state $\left(E_{b}>0\right)$ that is below the scattering threshold when $v_{m}>0$. (c) For $v_{m}<0$, there is a quasibound state in the continuum with energy $-E_{b}$ above the threshold. The scattering energy of two fermions extends to $2 E_{F}$ in a degenerate Fermi gas.

two fermions and is given by

$$
\begin{aligned}
\Pi_{m}\left(\mathbf{q}, i v_{n}\right)= & \frac{1}{V} \sum_{\mathbf{k}}\left\{k^{2}\left|Y_{1 m}(\hat{\mathbf{k}})\right|^{2}\right. \\
& \left.\times \frac{1-f\left(\xi_{\mathbf{k}+\mathbf{q} / 2}\right)-f\left(\xi_{-\mathbf{k}+\mathbf{q} / 2}\right)}{\xi_{\mathbf{k}+\mathbf{q} / 2}+\xi_{-\mathbf{k}+\mathbf{q} / 2}-i v_{n}}\right\},
\end{aligned}
$$

where $f(\xi)=[\exp (\beta \xi)+1]^{-1}$ is the Fermi distribution function. A direct evaluation of $\Pi_{m}$ shows that it is divergent and requires renormalization. This can be achieved together with the renormalization of $\Omega_{0}^{\mathrm{M}}$ by noticing that

$$
\begin{aligned}
& \ln \left[1+g_{m}^{2} \Pi_{m} G_{0}^{\mathrm{M}}\right] \\
& \quad=\ln g_{m}^{2}+\ln G_{0}^{\mathrm{M}}+\ln \left[g_{m}^{-2}\left(G_{0}^{\mathrm{M}}\right)^{-1}+\Pi_{m}\right] .
\end{aligned}
$$

The first term is a constant and can be neglected. The second term, when integrated in Eq. (6), cancels precisely $\Omega_{0}^{\mathrm{M}}$. One is thus left with the last term, which, by means of the renormalization conditions, Eqs. (3) and (4), reduces to

$$
\Omega \equiv \Omega_{0}^{\mathrm{F}}+\widetilde{\Omega}_{\mathrm{int}}=\Omega_{0}^{\mathrm{F}}-\sum_{m, \mathbf{q}} \int \frac{d z}{\pi} \frac{1}{e^{\beta z}-1} \delta_{m}(\mathbf{q}, z),
$$

an expression of identical form as that for the single channel model [32]. Here $\delta_{m}(\mathbf{q}, z)$ is the $p$-wave scattering phase shift and is given by $\delta_{m}(\mathbf{q}, z)=-\arg \left[\Gamma_{m}^{-1}(\mathbf{q}, z)\right]$, where the vertex function is given by

$$
\Gamma_{m}^{-1}(\mathbf{q}, z)=\frac{M^{2}}{4 \pi R_{m}} \bar{z}+\frac{M}{4 \pi v_{m}}+\Pi_{m}^{r}(\mathbf{q}, z) .
$$


The renormalized polarization $\Pi_{m}^{r}(\mathbf{q}, z)$ is

$$
\Pi_{m}^{r}(\mathbf{q}, z)=\Pi_{m}(\mathbf{q}, z)-\frac{M}{V} \sum_{\mathbf{k}} 1-\frac{M \bar{z}}{2 V} \sum_{\mathbf{k}} \frac{1}{\epsilon_{\mathbf{k}}},
$$

where $\bar{z}=z-\epsilon_{\mathbf{q}} / 2+2 \mu$.

\section{A. Analytic structure of the vertex function}

The structure of the vertex function $\Gamma_{m}$ can be analyzed most easily at high temperature where one can neglect the Fermi distribution factors in Eq. (8). In that limit we find analytically $\Gamma_{m}^{-1}(\mathbf{q}, z)=M^{2} /\left(4 \pi R_{m}\right) \bar{z}+M /\left(4 \pi v_{m}\right)+$ $M^{5 / 2} /(4 \pi)(-\bar{z})^{3 / 2}$. For $v_{m}>0$, this leads to two bound states where $\bar{z}<0$. In the resonant regime where the dimensionless parameter $\left|k_{F}^{3} v_{m}\right| \gg 1$ and the correspondingly $R_{m}$ assumes its natural scale in low-energy scattering $\left(k_{F} R_{m} \ll 1\right)$, the two poles of $\Gamma_{m}(\mathbf{q}, z)$ are given by $\bar{z}_{1}=-R_{m} /\left(M v_{m}\right)<0$ and $\bar{z}_{2}=-1 /\left(M R_{m}^{2}\right)$. This means that $\bar{z}_{2}$ corresponds to a very deeply bound state which lies outside the validity of effective range expansion used in our work. In fact, it is known that $\bar{z}_{2}$ corresponds to the ghost field where the normalization for such a state becomes negative [33,34]. As a result, in the implementation of NSR calculation, we should neglect the $\bar{z}_{2}$ pole of $\Gamma_{m}^{-1}$, which is allowed if one is only interested in the low-energy properties of the system.

The other pole $\bar{z}_{1}=-R_{m} /\left(M v_{m}\right)<0$ corresponds to a weakly bound state when $v_{m}>0$. This will be referred to as the BEC side of the resonance [see Fig. 1(b)]. As one tunes across the resonance $\left(v_{m}= \pm \infty\right)$ towards the BCS side $\left(v_{m}<0\right)$, the bound state emerges above zero energy and becomes a quasibound state because of the $p$-wave centrifugal barrier [see Fig. 1(c)]. In this case, $\bar{z}_{1}$ acquires a small imaginary part, $\propto R_{m} /\left(M v_{m}\right) \sqrt{R_{m}^{3} / v} \ll R_{m} /\left(M v_{m}\right)$, because of its couplings to the scattering fermions. It is interesting to note that distinct from the $s$-wave case, where the pole is purely imaginary in the BCS side of the resonance, in the $p$-wave case, it has a dominating real part. This is because of the $p$-wave centrifugal barrier which allows for the existence of a quasibound $p$-wave dimer state within the centrifugal barrier. For $k_{\mathrm{B}} T \ll\left|\bar{z}_{2}\right|$, it is then crucial to take into account the contribution from $\bar{z}_{1}$ pole, whose energy we denote as $E_{b}^{m}=R_{m} /\left(M v_{m}\right)$ below.

In the high-temperature limit $E_{F} \ll k_{\mathrm{B}} T \ll\left|\bar{z}_{2}\right|, \widetilde{\Omega}_{\text {int }}$ can be expanded to lowest order in fugacity $\exp (\beta \mu)$. We can write $\delta_{m}(\mathbf{q}, z)=\delta_{m}^{\mathrm{M}}(\mathbf{q}, z)+\delta_{m}^{\mathrm{F}}(\mathbf{q}, z)$ in Eq. (10), where $\delta_{m}^{\mathrm{M}}(\mathbf{q}, z)$ arises from the molecular pole $\bar{z}_{1}$ and $\delta_{m}^{\mathrm{F}}(\mathbf{q}, z)$ for the scattering fermions. In the absence of the many-body medium effects [neglecting the Fermi distribution function in Eq. (8)], one can reduce Eq. (10) to the standard virial expansion results where $\delta_{m}^{\mathrm{M}}(\mathbf{q}, z)$ gives the contribution from bound molecules and $\delta_{m}^{\mathrm{F}}(\mathbf{q}, z)$ for the scattering fermions [32]. At temperature $T>$ $T_{c}$ and for a given set of scattering parameters $\left\{v_{m}, R_{m}\right\}$, one can solve for the thermodynamic potential in Eq. (10) together with the number equation $N=N_{\mathrm{F}}+2 N_{\mathrm{B}}=-\partial \Omega / \partial \mu$.

\section{ENERGETICS AND UNIVERSAL EQUATION OF STATE}

In experiment, the effective range $R_{m}$ is approximately a constant around resonance and, furthermore, independent of magnetic quantum number $m$. As a result, we set $R_{m}=R$ in the following for simplicity. On the other hand, the resonances

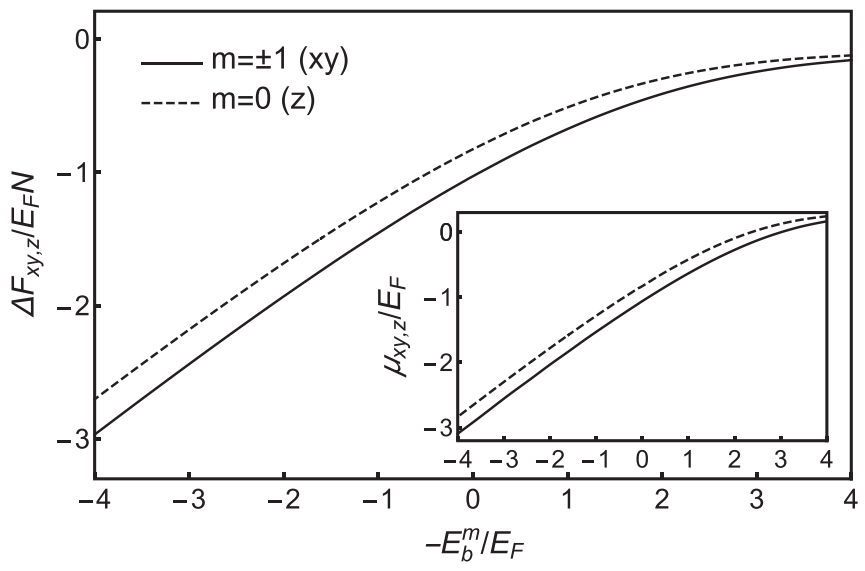

FIG. 2. Relative free energy $\Delta F_{x y, z}=F_{x y, z}-F_{x y, z}^{0}$ as a function of $-E_{b}^{m} / E_{F}$ close to the $x y$ and $z$ resonances at $k_{\mathrm{B}} T=0.8 E_{F}$. Here $F_{x y, z}^{0}$ is the corresponding free energy for noninteracting system. In our calculation, we have set $k_{F} R=0.04$, appropriate to the experiment. $F_{x y}$ (solid line) is always smaller than that of the $F_{z}$ (dashed line) due to multiple molecular bound states. Inset shows the chemical potential $\mu_{x y, z}$ as a function of $-E_{b}^{m} / E_{F}$ for the same set of parameters.

for $m=0$ ( $z$ resonance $)$ and $m= \pm 1$ ( $x y$ resonance) are split due to magnetic dipole-dipole interactions [9]. In the vicinity of the $x y$ resonance, there are two possible molecular states in the closed channel, while for the $z$ resonance, there is only one. In ${ }^{40} \mathrm{~K}$ experiments, these two resonances are well separated and do not interfere with each other which allows us to investigate the properties of the gas for the $x y$ and $z$ resonance separately.

The free energy $F_{x y, z} \equiv \Omega_{x y, z}+\mu_{x y, z} N$ of the system can be written in the following universal form around the $x y$ and $z$ resonances: $F_{x y, z}=N E_{F} f_{x y, z}\left(k_{F} R,-E_{b}^{m} / E_{F}, k_{\mathrm{B}} T / E_{F}\right)$. In this work, we focus on the dependences of $f_{x y, z}$ on $E_{b}^{m} / E_{F}$, assuming $k_{F} R=0.04$, a typical experimental value. Note that the scaling form for $F_{x y, z}$ works for both normal and superfluid phases. For normal state, we calculate the scaling functions $f_{x y, z}$ within NSR for $k_{\mathrm{B}} T=0.8 E_{F}$ (see Fig. 2). For both resonances, the free energy decreases monotonically from the BCS side to the BEC side. Throughout the crossover, $f_{x y}<f_{z}$ since there exist two molecular bound states for $x y$ resonance and this lowers its free energy. In fact, the difference $\left|f_{x y}-f_{z}\right|$ increases as one moves towards the BEC limit. Close to resonance, the reduction of free energy is of the order of Fermi energy, indicating strong $p$-wave interactions.

\section{V. p-WAVE CONTACTS}

One of the most exciting aspects of the $p$-wave resonantly interacting Fermi gas is the existence of an extended set of universal relations involving the $p$-wave contacts. As in the $s$-wave case, the $p$-wave contacts determine the universal properties of the system including their response to external radio-frequency field, which has been utilized to measure the $p$-wave contacts in recent experiment [14]. Theoretically, one can calculate the values of the relevant contacts using the adiabatic theorems, which relate them to the variation of the 

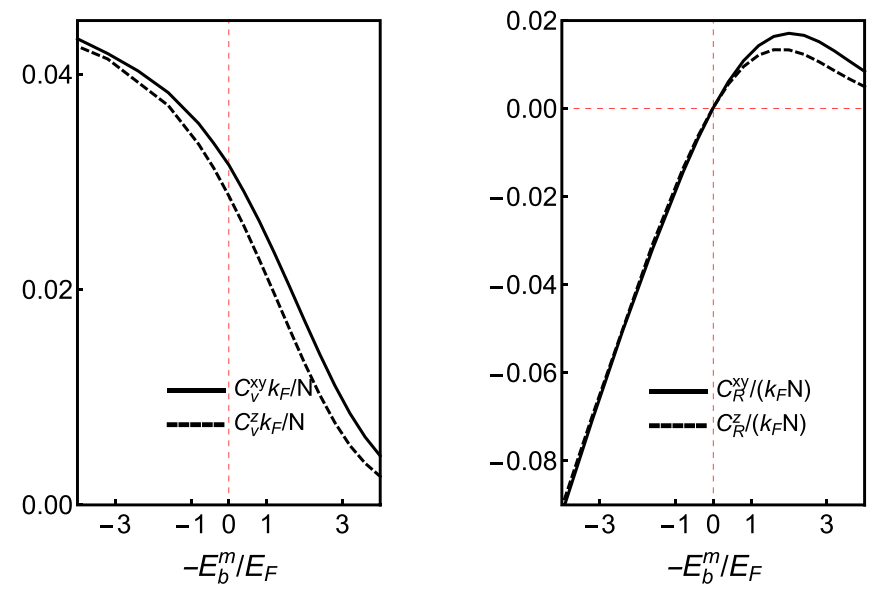

FIG. 3. Contacts $C_{v}^{x y, z}$ (left) and $C_{R}^{x y, z}$ (right) as a function of $-E_{b}^{m} / E_{F}$ for $k_{\mathrm{B}} T=0.8 E_{F}$ and $k_{F} R=0.04$. $C_{v}^{x y}$ (solid line) and $C_{v}^{z}$ (dashed line) decrease monotonically from the BEC to the BCS side with $C_{v}^{z}$ always smaller than $C_{v}^{x y}$. On the other hand, both $C_{R}^{x y}$ (solid line) and $C_{R}^{z}$ (dashed line) vanish at resonance $v= \pm \infty$ and depend on $-E_{b}^{m} / E_{F}$ nonmonotonically. The magnitude of $C_{R}^{z}$ is always smaller than $C_{R}^{x y}$.

free energy with respect to the scattering parameters:

$$
\left.\frac{\partial F}{\partial v_{z}^{-1}}\right|_{R, T}=-\frac{C_{v}^{z}}{2 M},\left.\quad \frac{\partial F}{\partial R^{-1}}\right|_{v_{z}, T}=-\frac{C_{R}^{z}}{2 M},
$$

and the similar equation for the $x y$ resonance.

In Fig. 3, we show the calculated values of $C_{v}^{x y, z}$ and $C_{R}^{x y, z}$ within NSR for $k_{F} R=0.04$ and $k_{\mathrm{B}} T=0.8 E_{F} \cdot C_{v}^{x y, z}$ is monotonically decreasing as a function of $-E_{b}^{m} / E_{F}$ from the BCS to BEC side and is always positive, consistent with the general requirement [16]. $C_{v}^{x y}$ is always greater than $C_{v}^{z}$, indicating a stronger dependence of $F_{x y}$ on $-E_{b}^{m} / E_{F}$ than $F_{z}$ due to multiple bound states in the closed channel. On the other hand, $C_{R}^{x y, z}$ shows nonmonotonic behavior as a function of $-E_{b}^{m} / E_{F}$, with maximal value always achieved in the BCS side of the resonance. Note that even though $C_{R}^{x y, z}$ changes sign across the resonance and vanishes when $v= \pm \infty$, the magnitude of $C_{R}^{x y}$ is always larger than $C_{R}^{z}$, consistent with stronger interactions around the $x y$ resonance.

One interesting aspect of a narrow $\left(k_{\mathrm{F}} R_{m} \ll 1\right) p$-wave resonance is that the quasibound dimer state has a long lifetime especially close to resonance $\left(R_{m}^{3} / v_{m} \ll 1\right)$. The small imaginary part, which also sets the scale for the fermiondimer coupling, thus suggests that the coupling between them is small and one may, to a very good approximation, treat fermions and dimers as two-independent components. Since within the NSR scheme, we did not take into account the direct fermion interactions, this means that one may be able to understand the NSR calculation in terms of the fermion-dimer mixture model. Recall that for a collection of noninteracting $p$-wave dimers with binding energy $R_{m} / M v_{m}, C_{v, m}=2 R_{m}$ and $C_{R, m}=-2 R_{m}^{2} / v_{m}$ [14], this means that $C_{R, m} / C_{v, m}=$ $-R_{m} / v_{m}$. Or in dimensionless form, we have

$$
\frac{C_{R, m} / k_{\mathrm{F}}}{C_{v, m} k_{\mathrm{F}}}=-\frac{1}{2} \frac{E_{b}^{m}}{E_{\mathrm{F}}} .
$$
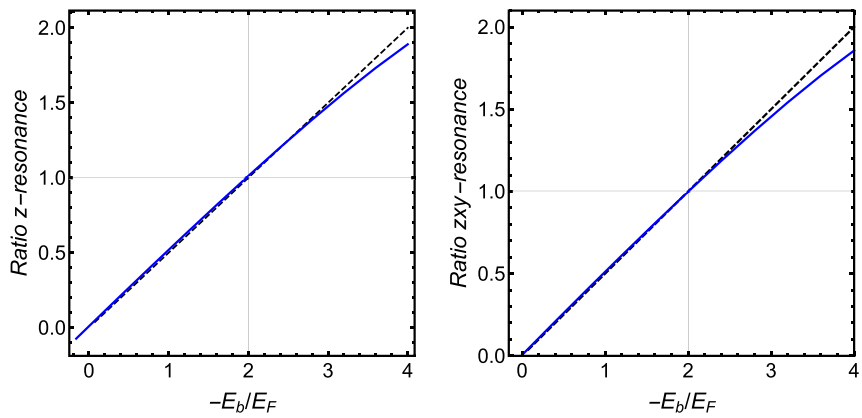

FIG. 4. Comparison with fermion-dimer mixture model shows that, in the resonance region, the prediction of NSR tracks closely that of the mixture model. Blue lines shows the NSR calculation and black dashed line indicates the prediction of mixture model, Eq. (14).

Indeed, if we plot the dimensionless ratio as in Fig. 4, we find that in the resonance regime, the linear relation is very well captured by the fermion-dimer mixture model except when the quasibound state moves out of the fermion continuum $\left(\sim 2 E_{\mathrm{F}}\right)$.

\section{SUPERFLUID TRANSITION TEMPERATURES}

Near a broad $s$-wave Feshbach resonance, superfluidity is the most robust at unitarity: the coherence length is shortest and the critical current is largest [12]. Near a $p$-wave resonance, the pairing symmetry is richer [29,35-38], and additional internal structure breaks scale invariance. In our formulation, apart from the splitting of resonance of $m= \pm 1(x y)$ and $m=0(z)$, the vertex function $\Gamma_{m}$ is diagonal in $m$. This means that close to $x y$ resonance around $T_{c}$, the superfluid order parameter is of the axial form, with gap function $\Delta_{\mathbf{k}}$ having the symmetry $Y_{1 \pm 1}(\hat{k})$ or their superposition. The detailed form cannot be obtained from our calculation within NSR. It is known that, however, the ground-state order parameter should be of the pure $Y_{1 \pm 1}(\hat{k})$ form [35]. Close to the $z$ resonance, the order parameter is the standard polar form, for which $\Delta_{\mathbf{k}} \propto k_{z}$. Using the Thouless criterion, we can write the equation for $T_{c}$ close to the $z$ resonance as $\Gamma_{z}^{-1}(\mathbf{0}, 0)=0$, or explicitly

$$
\frac{M^{2} \mu}{2 \pi R}+\frac{M}{4 \pi v}+\Pi_{z}^{r}(\mathbf{0}, 0)=0,
$$

and similar equation determining the $T_{c}$ for $x y$ resonance by replacing $\Pi_{z}^{r}$ with $\Pi_{x y}^{r}$.

In Fig. 5, we show the calculated critical temperature for the $x y$ and $z$ resonance. For the $x y$ resonance, the critical temperature is always lower than that for the $z$ resonance. This is because, for the $x y$ resonance, there are two molecular states in the closed channel which reduce the quantum degeneracy of the system. This is particularly evident in the BEC limit where all $N / 2$ bosons are divided between two molecular states (with $m \pm 1$, each with number $N / 4$ and density $n / 4$ ), so the corresponding critical temperature for BEC is given by $T_{c}^{x y} / T_{F}=0.086$. For the $z$ resonance, there is only one molecular state with $m=0$ and the corresponding number density is $N / 2 V=n / 2$. This gives a higher critical temperature $T_{c}^{z} / T_{F}=0.137$. These values are also consistent with the calculated $T_{c}$ via NSR in the BEC side. The general behavior of $T_{c}$ in our calculation is consistent with that of Ref. [39], where, 


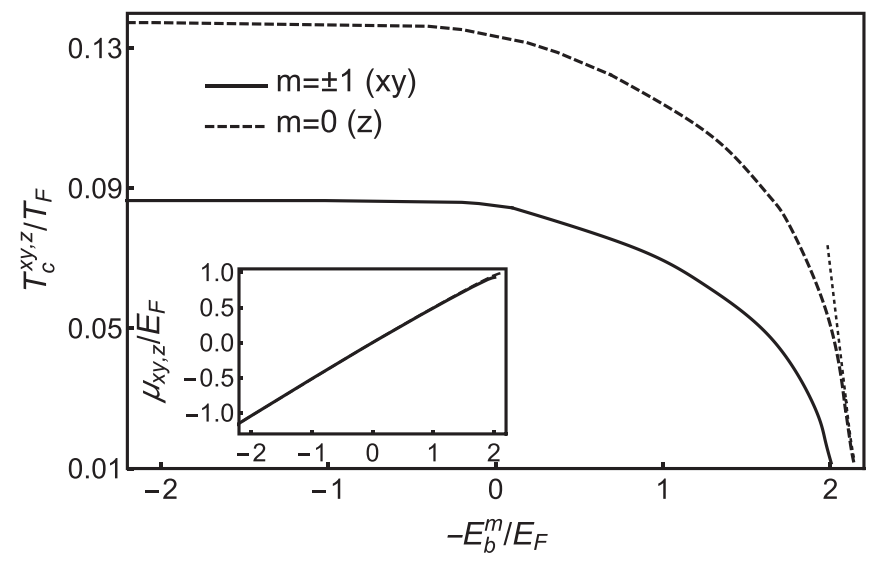

FIG. 5. Critical temperature $T_{c}$ as a function of $-E_{b}^{m} / E_{F}$ for the $x y$ (solid line) and $z$ resonances (dashed line). The dotted line is the asymptotic $T_{c}$ in the BCS limit for the $z$ resonance given by Eq. (16). Inset shows the respective chemical potentials for the $x y$ and $z$ resonances.

however, the dependence of $T_{c}$ on the low-energy scattering parameters $\left\{v_{m}, R_{m}\right\}$ is implicit since it uses the bare coupling constants with a cutoff.

In the BCS limit, $v \rightarrow 0^{-}$, the attractive interaction is very weak and $T_{c}$ becomes very small. In this limit, Eq. (15) simplifies considerably and one can obtain the asymptotic value of $T_{c}$ as

$$
T_{c}=\frac{8 \gamma \tilde{\mu}}{\pi} T_{F} \exp \left[-\frac{8}{3} \tilde{\mu}^{\frac{3}{2}}\right] \exp \left[\frac{\pi \tilde{\mu}}{2 k_{F} R}+\frac{\pi}{2 k_{F}^{3} v}\right],
$$

where $\gamma=1.78107$ is the exponential of the Euler constant and $\tilde{\mu}=\mu / E_{F}$. Note the standard exponential dependences on the $p$-wave interaction parameter, the scattering volume $v$, similar to the $s$-wave case. In addition, it should be noted that the dependence on the effective range $R$ is also nonanalytic, indicating the importance of finite range corrections in a dilute $p$-wave superfluid. Setting $\tilde{\mu}=1$ in Eq. (16), one can equivalently write [40]

$$
T_{c}=\frac{8 \gamma}{\pi} T_{F} \exp \left[-\frac{8}{3}\right] \exp \left[\frac{\pi}{2 k_{F} R}\left(1-\frac{E_{b}}{2 E_{F}}\right)\right],
$$

showing that $T_{c}$ changes rapidly around $E_{b} / E_{F} \approx 2$ when the quasibound $p$-wave state moves out of the scattering continuum.
At the critical temperature $T=T_{c}$, the corresponding chemical potential $\mu_{x y, z}$ as a function of $E_{b}^{m} / E_{F}$ is shown in the inset of Fig. 5. The chemical potential $\mu_{x y, z}$ is approximately half of the binding energy $E_{b}^{m}$ and is thus linear in $E_{b}^{m}$ over a wide range of interaction, but it quickly approaches $E_{F}$ around $E_{b}^{m} / E_{F} \approx 2$ in the BCS limit. When $\mu=0$, there is a simple relation between the critical temperature and the scattering volume $v$

$$
\left.\frac{1}{k_{F}^{3} v}\right|_{\mu=0}=\frac{2-\sqrt{2}}{2 \sqrt{\pi}} \zeta(3 / 2)\left(\frac{T_{c}}{E_{F}}\right)^{3 / 2},
$$

where $T_{c}$ is the critical temperature at $\mu=0$. We note that this relation is independent of the effective range $R$.

\section{CONCLUSION}

In this article, we studied the normal-state properties of a resonantly interacting $p$-wave Fermi gas. The universal equation of state, the $p$-wave contacts, and the superfluid transition temperatures $T_{c}$ are obtained using a two-channel formulation. We show that, for the resonance with $m= \pm 1$, our estimation of the transition temperatures using actual experimental parameters is quite encouraging experimentally. We also show that due to the small effective range $k_{\mathrm{F}} R_{m} \ll 1$, the prediction of the NSR calculation is similar to that of the fermion-dimer mixture model.

There remain further important theoretical questions to be investigated such as the analogous Gor'kov-MelikBarkhudarov correction to $T_{c}$ from medium polarizations [41]. It might be also useful to include the residue fermion-fermion interactions to see how they modify the fermion-dimer mixture model. Experimentally, it would be very useful to find $p$-wave resonances with a larger effective range and investigate the many-body effects arising from stronger fermion-dimer couplings.

\section{ACKNOWLEDGMENTS}

We thank Boyang Liu, Shanshan Ding, and Joseph Thywissen for helpful discussions. This work is supported by Hong Kong Research Grants Council, General Research Fund, Grant No. HKU 17318316, CRF, Grant No. HKUST3/CRF/13G, and the Croucher Foundation under the Croucher Innovation Award.
[1] C. A. Regal, C. Ticknor, J. L. Bohn, and D. S. Jin, Phys. Rev. Lett. 90, 053201 (2003).

[2] J. Zhang, E. G. M. van Kempen, T. Bourdel, L. Khaykovich, J. Cubizolles, F. Chevy, M. Teichmann, L. Tarruell, S. J. J. M. F. Kokkelmans, and C. Salomon, Phys. Rev. A 70, 030702(R) (2004).

[3] K. Günter, T. Stöferle, H. Moritz, M. Köhl, and T. Esslinger, Phys. Rev. Lett. 95, 230401 (2005).

[4] C. H. Schunck, M. W. Zwierlein, C. A. Stan, S. M. F. Raupach, W. Ketterle, A. Simoni, E. Tiesinga, C. J. Williams, and P. S. Julienne, Phys. Rev. A 71, 045601 (2005).
[5] J. P. Gaebler, J. T. Stewart, J. L. Bohn, and D. S. Jin, Phys. Rev. Lett. 98, 200403 (2007).

[6] J. Fuchs, C. Ticknor, P. Dyke, G. Veeravalli, E. Kuhnle, W. Rowlands, P. Hannaford, and C. J. Vale, Phys. Rev. A 77, 053616 (2008).

[7] Y. Inada, M. Horikoshi, S. Nakajima, M. Kuwata-Gonokami, M. Ueda, and T. Mukaiyama, Phys. Rev. Lett. 101, 100401 (2008).

[8] T. Nakasuji, J. Yoshida, and T. Mukaiyama, Phys. Rev. A 88, 012710 (2013).

[9] C. Ticknor, C. A. Regal, D. S. Jin, and J. L. Bohn, Phys. Rev. A 69, 042712 (2004). 
[10] F. Chevy, E. G. M. van Kempen, T. Bourdel, J. Zhang, L. Khaykovich, M. Teichmann, L. Tarruell, S. J. J. M. F. Kokkelmans, and C. Salomon, Phys. Rev. A 71, 062710 (2005).

[11] M. Waseem, Z. Zhang, J. Yoshida, K. Hattori, T. Saito, and T. Mukaiyama, J. Phys. B: At. Mol. Opt. Phys. 49, 204001 (2016).

[12] The BCS-BEC Crossover and the Unitary Fermi Gas, edited by W. Zwerger (Springer-Verlag, Berlin, 2011).

[13] J. Levinsen, N. R. Cooper, and V. Gurarie, Phys. Rev. A 78, 063616 (2008).

[14] C. Luciuk, S. Trotzky, S. Smale, Z. Yu, S. Zhang, and J. H. Thywissen, Nat. Phys. 12, 599 (2016).

[15] S. M. Yoshida and M. Ueda, Phys. Rev. Lett. 115, 135303 (2015).

[16] Z. Yu, J. H. Thywissen, and S. Zhang, Phys. Rev. Lett. 115, 135304 (2015); 117, 019901 (2016).

[17] M. He, S. Zhang, H. M. Chan, and Q. Zhou, Phys. Rev. Lett. 116, 045301 (2016).

[18] S.-G. Peng, X.-J. Liu, and H. Hu, Phys. Rev. A 94, 063651 (2016).

[19] X. Cui and H. Dong, Phys. Rev. A 94, 063650 (2016).

[20] X. Cui, Phys. Rev. A 94, 043636 (2016).

[21] Y.-C. Zhang and S. Zhang, Phys. Rev. A 95, 023603 (2017)

[22] S. Tan, Ann. Phys. (NY) 323, 2952 (2008).

[23] E. Braaten and L. Platter, Phys. Rev. Lett. 100, 205301 (2008).

[24] S. Zhang and A. J. Leggett, Phys. Rev. A 79, 023601 (2009).

[25] F. Werner, L. Tarruell, and Y. Castin, Eur. Phys. J. B 68, 410 (2009).
[26] S.-L. Zhang, M. He, and Q. Zhou, Phys. Rev. A 95, 062702 (2017).

[27] S. M. Yoshida and M. Ueda, Phys. Rev. A 94, 033611 (2016).

[28] R. Qi, arXiv:1606.03299.

[29] V. Gurarie and L. Radzihovshy, Ann. Phys. (NY) 322, 2 (2007).

[30] J. Levinsen, P. Massignan, F. Chevy, and C. Lobo, Phys. Rev. Lett. 109, 075302 (2012).

[31] P. Nozières and S. Schmitt-Rink, J. Low Temp. Phys. 59, 195 (1985).

[32] C. J.Pethick and H. Smith, Bose-Einstein Condensation in Dilute Gases, 2nd ed. (Cambridge University Press, Cambridge, UK, 2008).

[33] Y. Nishida, Phys. Rev. A 86, 012710 (2012).

[34] E. Braaten, P. Hagen, H.-W. Hammer, and L. Platter, Phys. Rev. A 86, 012711 (2012).

[35] T.-L. Ho and R. B. Diener, Phys. Rev. Lett. 94, 090402 (2005).

[36] C.-H. Cheng and S. K. Yip, Phys. Rev. Lett. 95, 070404 (2005).

[37] V. Gurarie, L. Radzihovsky, and A. V. Andreev, Phys. Rev. Lett. 94, 230403 (2005).

[38] C.-H. Cheng and S.-K. Yip, Phys. Rev. B 73, 064517 (2006).

[39] Y. Ohashi, Phys. Rev. Lett. 94, 050403 (2005).

[40] M. Iskin and C. A. R. Sa de Melo, Phys. Rev. Lett. 96, 040402 (2006).

[41] L. P. Gorkov and T. K. Melik-Barkhudarov, Sov. Phys. JETP 13, 1018 (1961). 